\title{
Reluctance Machine for a Hollow Cylinder Flywheel
}

\author{
Magnus Hedlund *, Tobias Kamf, Juan de Santiago, Johan Abrahamsson and Hans Bernhoff \\ Division for Electricity, Uppsala University, Lägerhyddsvägen 1, 75237 Uppsala, Sweden; \\ tobias.kamf@angstrom.uu.se (T.K.); juan.santiago@angstrom.uu.se (J.d.S.); \\ johan.abrahamsson@angstrom.uu.se (J.A.); hans.bernhoff@angstrom.uu.se (H.B.) \\ * Correspondence: magnus.hedlund@angstrom.uu.se; Tel.: +46-18-471-5804
}

Academic Editor: K.T. Chau

Received: 28 October 2016; Accepted: 28 February 2017; Published: 7 March 2017

\begin{abstract}
A hollow cylinder flywheel rotor with a novel outer rotor switched reluctance machine (SRM) mounted on the interior rim is presented, with measurements, numerical analysis and analytical models. Practical experiences from the construction process are also discussed. The flywheel rotor does not have a shaft and spokes and is predicted to store $181 \mathrm{Wh} / \mathrm{kg}$ at ultimate tensile strength (UTS) according to simulations. The novel SRM is an axial flux machine, chosen due to its robustness and tolerance for high strain. The computed maximum tip speed of the motor at UTS is $1050 \mathrm{~m} / \mathrm{s}$. A small-scale proof-of-concept electric machine prototype has been constructed, and the machine inductance has been estimated from measurements of voltage and current and compared against results from analytical models and finite element analysis (FEA). The prototype measurements were used to simulate operation during maximal speed for a comparison towards other high-speed electric machines, in terms of tip speed and power. The mechanical design of the flywheel was performed with an analytical formulation assuming planar stress in concentric shells of orthotropic (unidirectionally circumferentially wound) carbon composites. The analytical approach was verified with 3D FEA in terms of stress and strain.
\end{abstract}

Keywords: flywheel energy storage (FES); hollow cylinder flywheel; reluctance machine; high-speed machines

\section{Introduction}

A new era of high-speed flywheel energy storage (FES) started with the advent of carbon composite flywheels, a technology that has become more viable due to a trend of decreasing costs of power electronics, position sensors (for magnetic bearings) and microprocessors [1]. The commercial potential for FES systems has recently gained attention in various applications. In racing sports, an FES powered the winning car of the legendary Le Mans race for several years. Flywheels have been mounted on inner city hybrid buses to increase fuel efficiency. The technology has also been proven to enable the use of intermittent renewable energy sources, for example by stabilizing the electric grid at remote wind farms, allowing for better utilization of transmission lines. Improving the capacity factor of power lines is important in all applications when the cost of the transmission itself is a significant cost of the installation, such as for offshore wind power or wave power parks. FESs are used to provide fast regulation power for maintaining the grid frequency, decreasing the use of inefficient coal plants with low ramp rates for the task. On a distribution grid and end customer level, FESs are used to improve power quality for industries and data centers [1].

Modern, electrified flywheels are mainly composed of steel, aluminum, titanium, carbon and glass fiber composites, which are all readily available in bulk volumes. Energy is stored when the electric motor/generator accelerates the flywheel, and energy is released when the electric machine acts as a generator and slows down the flywheel. Flywheels compete with storage technologies, such as 
batteries and electrolytically double-layered capacitors (EDLCs, popularly known as supercapacitors) in applications that require a large number of charge-discharge cycles with high specific power. Flywheels excel when compared against batteries in terms of specific power and cycling fatigue. Comparing costs is harder however, since the latter is mass-produced, but the former is not. An estimate can be computed based on the knowledge that for rotating electrical machines, raw materials comprise about $60 \%-70 \%$ of the cost [2]. GKN Hybrid Power (Worcestershire, UK) reported that the raw material cost of a standard internal combustion engine is about $685 \mathrm{USD}$, compared to their $30 \mathrm{~kW} / 111 \mathrm{Wh}$ flywheel for automotive applications, which costs about 723 USD [3]. The resulting cost would then be about 1100 USD, or 10,000 USD/kWh, which is about 30-times more expensive than Li-ion batteries [4]. On the other side, a high-performance Li-ion battery can only use $3 \%$ of its capacity to withstand $10^{6}$ charge-discharge cycles (which FESs and EDLCs are typically rated for), during moderate discharge rates $(\leq 1 C)[5]$. It should also be noted, however, that a flywheel excels at delivering power while retaining a good cycle life. A battery topology with a system specific energy of $200 \mathrm{Wh} / \mathrm{kg}$ (including cooling system, etc.) with a weight of $0.6 \mathrm{~kg}$ would match the GKN flywheel (in terms of energy capacity) mentioned above, not taking into account cycling properties. Assuming a requirement of $10^{6}$ cycles, the battery system weight would be increased to $20 \mathrm{~kg}$, and with a discharge rate of $1 \mathrm{C}$, the maximum power is then only $4 \mathrm{~kW}$. To meet the power requirements combined with cycling properties, the system weight would have to be increased to $150 \mathrm{~kg}$, which is 10-times heavier than a modern flywheel system [1]. Li-ion batteries can be and are currently used in F1 racing at higher power levels, but the battery life becomes very limited.

The specific energy of state-of-the-art automotive FESs (GKN Hybrid Porsche GT3R Flywheel [6]) is 2.7-times larger than state-of-the-art automotive EDLCs (Maxwell 125V Heavy Transportation Module [7]), while retaining a specific power 1.8-times larger [1]. However, FESs are considered to be systems of high complexity, when compared to batteries and supercapacitors. Increasing the specific energy of FESs is important since more end applications become feasible technically and viable economically.

\section{Optimizing Specific Energy}

The specific energy $e$ grows quadratically with radius $r$ from a fixed rotational axis, as can be seen by inspecting the kinetic energy $E$ in a rotating body per unit mass $m$ (with a rotational velocity of $\omega$ ):

$$
e=\frac{E}{m}=\frac{J \omega^{2}}{2 m}=\frac{\omega^{2}}{2 m} \int r^{2} d m,
$$

where the moment of inertia around a fixed axis $J$ is expanded into its integral definition. The formula essentially states the three ways to optimize for specific energy:

- Increase rotational speed;

- Decrease mass;

- Distribute mass closer to the rim.

The advent of carbon composites has greatly progressed flywheel development in terms of the first two bullet points above. This paper aims to provide progress in the field with regards to the third option by examining an electric machine with properties suitable for hollow cylinder flywheels.

The paper is divided into three parts: Firstly, hollow cylinder flywheels are introduced, and a theoretical rotor design is presented. Secondly, a switched reluctance machine (SRM) for the aforementioned rotor is presented with measurements and simulations. Thirdly, simulations based on the above measurements are used to compare the SRM with other high-speed machines.

\section{Integrated Hollow Flywheels}

The connection of a composite flywheel rim to a shaft is an issue well addressed in the technical literature [8-24]. The most common class of solutions can be denoted strain-matching and can be 
traced far back before the unidirectionally-wound composite flywheel era [13] where the spokes or hub are made of materials and/or shapes that strain in a similar fashion as the rim when exposed to centrifugal forces. Both metallic [14-16] and composite [18,19] solutions exist. Another category is the conical hubs, where a conical metal interface yields the elastic properties needed to compensate for the rim expansion [9-11]. Composite solutions also exist [12,22]. A less common category is the flexible rim in which stiff composite spokes holding an elliptical rim at standstill will deform the rim into a circle at nominal strain and speed $[19,20]$. Other interesting solutions include: having flexible composite spokes in which the rim and hub are connected with continuous fibers [23]; or having a stiff metal hub connect to a composite rim with a high-strain interface made of PVC, nylon or brass [24].

Recently, flywheels with no shaft or spokes have begun to surface. Instead, the electromagnetic actuators (electric machine and magnetic bearings) reside on the inside of a composite rim. These hollow rotor solutions have been developed as a way to increase rotor specific energy and, thus, decrease material usage and decrease cost [21,25], where an industrial manufacturer claims a potential for an $8 \times$ decrease in cost $[26,27]$. The decreasing cost of magnetic bearings (in terms of sensors, feedback electronics and power electronics) has made the hollow cylinder technology increasingly feasible. However, a shaft-less design demands more in terms of material strength for the electromagnetically-active parts used for accelerating and suspending the rotor, since these must be placed closer to the rim where the centrifugal forces are larger. Hollow flywheels also face heating issues, since the whole rotor must be enclosed in low pressure due to high speeds, limiting the natural heat transport from the rotor to black-body radiation.

With maximal rim speeds in excess of one $\mathrm{km}$ per second [28], a relatively thin rimmed hollow rotor implies high relative velocities also on the inside of the rotating cylindrical shell. This in turn implies that the mechanical power transmission and mechanical bearings commonly used [1] are infeasible, suggesting the use of magnetic bearings, which have excellent high-relative speed characteristics $[8,29]$.

Below, we will analyze the strain-problem on the inside of a hollow composite rim, as well as show that the radial size of electromagnetically-active steel mounted on the inside of the composite rim is a major limiting factor to ultimate specific energy.

Note that the analysis below is not complete, since a practical design will always have a lower capacity than as defined by the specific energy at breaking. For example, in mass-production (especially in larger machines), it can be more cost effective to increase the rotor safety factor instead of increasing quality requirements for the carbon composite materials. Depending on the end application, the containment itself can impose limits on maximum realistic speed. In automotive applications, there is a trade off between rotor maximal speed and containment weight/strength.

Below, we derive plane stress equations for a long hollow cylinder flywheel and argue based on these that the electromagnetically-active material becomes a major limiting factor. The results are verified with an FEM simulation.

\subsection{Governing Equations}

\subsubsection{Isotropic Materials}

The radial and hoop stresses can be computed for a hollow cylinder with the following equations [30]:

$$
\left\{\begin{array}{l}
\sigma_{r}(r)=\frac{3+v}{8} \rho \omega^{2}\left(r_{o}^{2}+r_{i}^{2}-\frac{r_{o}^{2} r_{i}^{2}}{r^{2}}-r^{2}\right) \\
\sigma_{\theta}(r)=\frac{3+v}{8} \rho \omega^{2}\left(r_{o}^{2}+r_{i}^{2}+\frac{r_{o}^{2} r_{i}^{2}}{r^{2}}-\frac{1+3 v}{3+v} r^{2}\right)
\end{array}\right.
$$

where $\rho$ is the mass density, $v$ is Poisson's ratio, $r_{0}, r_{i}$ is the outer and inner radius of the rotor and $r$ is any radius in that interval. These equations are valid for any isotropic material, such as aluminum, steel or titanium, in a planar stress case. 


\subsubsection{Transversely Isotropic Materials}

Unidirectionally wound carbon fiber composites exhibit anisotropic material properties, which can be modeled as a transversely isotropic material in the $r z$-plane in a cylindrical coordinate system.

The corresponding stress equations can be seen below [8,31]:

$$
\left\{\begin{array}{ll}
\sigma_{r}= & C_{1} r^{-1-\mu}+C_{2} r^{-1+\mu}+\frac{\rho \omega^{2} r^{2}}{\mu^{2}-9}(3+\beta) \\
\sigma_{\theta}= & C_{1} \mu r^{-1-\mu}+C_{2} \mu r^{-1+\mu}+\frac{\rho \omega^{2} r^{2}}{\mu^{2}-9}\left(3 \beta+\mu^{2}\right)
\end{array},\right.
$$

where:

$$
\begin{gathered}
C_{1}=\frac{K\left(r_{i}^{3} r_{o}^{\mu}-r_{i}^{\mu} r_{o}^{3}\right)+P_{i} r_{o}^{\mu} r_{i}-P_{o} r_{i}^{\mu} r_{o}}{r_{i}^{2 \mu}-r_{o}^{2 \mu}}\left(r_{i} r_{o}\right)^{\mu}, \\
C_{2}=\frac{K\left(r_{o}^{\mu+3}-r_{i}^{\mu+3}\right)+P_{o} r_{o}^{\mu+1}-P_{i} r_{i}^{\mu+1}}{r_{i}^{2 \mu}-r_{o}^{2 \mu}},
\end{gathered}
$$

and:

$$
\begin{aligned}
& K=\frac{\rho \omega^{2}}{\mu^{2}-9}(3+\beta), \\
& \mu^{2}=\frac{E_{\theta}}{E_{r}} \frac{1-v_{z r} v_{r z}}{1-v_{z \theta} v_{\theta z}}, \\
& \beta=v_{\theta z} \frac{1+v_{z r}}{1-v_{z \theta} v_{\theta z}},
\end{aligned}
$$

where $E$ is the elastic modulus and $P$ is the pressure on the inner and outer surfaces. For a single hollow shell, $P$ is zero. In a situation where several shells are shrink-fitted onto each other (as discussed below), there are interface pressures in between these shells. Then, for two subsequent shells $m, n$, the following boundary condition applies:

$$
P_{o, m}=P_{i, n}
$$

together with a zero-interference constraint.

The stress equations can then be solved with numerical methods. For a multi-shell situation, a system of equations is formed. These equations have been shown to work well for quickly solving for stress [31], allowing for iterative testing of different designs. The equations can be used to approximate stress for segmented inner shells by assuming a low $E_{\theta}$. The final design is then verified in terms of both stress and strain in 3D FEA software.

\subsection{Practical Considerations}

The carbon fibers are unidirectionally tangentially wound within an epoxy matrix to attain maximal hoop tensile strength. The strength in radial and axial directions is dependent on the epoxy matrix, which is orders of magnitude weaker than the fibers. To alleviate radial stress limitations, several thin composite shells are shrink-fitted onto each other. The shrink-fit process (and also the matrix curing process) limits how much interface pressure can be attained between shells, which in turn limits the maximal speed [31]. To account for this in the design below, thermal expansion limitations were accounted for when computing interference pressure.

\subsection{Mechanical Simulation Results}

A hollow cylinder flywheel rotor design was found by iteratively solving the equations presented above for a multi-shell system. The final solution was then verified within the FEA software Solidworks, and the corresponding stresses and strains can be seen in Figure 1a. For the analytical equations, the steel was simulated as segmented by assuming a low $E_{\theta}$ (similar in approach to [31]), and in FEA, 
it was done by simulating a $5^{\circ}$ segment, as seen in Figure 1c. The rotor is composed of four shells. The innermost shell was constructed of laminated spring steel sheet metal with a stack thickness of $5.5 \mathrm{~mm}$, and the three outer shells are Toray T1000G (Toray CFA, Flower Mound, TX, USA)/epoxy composite material, with a total thickness of $10 \mathrm{~cm}$. The ultimate specific energy for this design is $181 \mathrm{Wh} / \mathrm{kg}$, at a rim speed of $1260 \mathrm{~m} / \mathrm{s}$, with the composite pushed close to its theoretical limits [32]. This value can be compared to $195 \mathrm{Wh} / \mathrm{kg}$, the highest ultimate specific energy flywheel rotor known to date [28], although that rotor had no electric machine (in contrast to this one). The hoop stress in the composite is increased due to the weight of the slit steel, which provides no hoop strength on its own. This sets an upper limit for steel thickness if optimizing for specific energy in a hollow rotor. On the other hand, the centrifugal forces within the composite rims increase with radius, causing tensile stress to build up, which can lead to delamination. Since the radial strength is much lower than the hoop strength for unidirectionally-wound composites, having steel on the inside can help keep the radial stress compressive (similar to [31]). The composite shells were press fitted. Friction forces between consecutive shells were assumed to be zero. It is thus seen that high specific energy can be attained in hollow steel/carbon composite rotors, but that steel thickness is an important parameter.

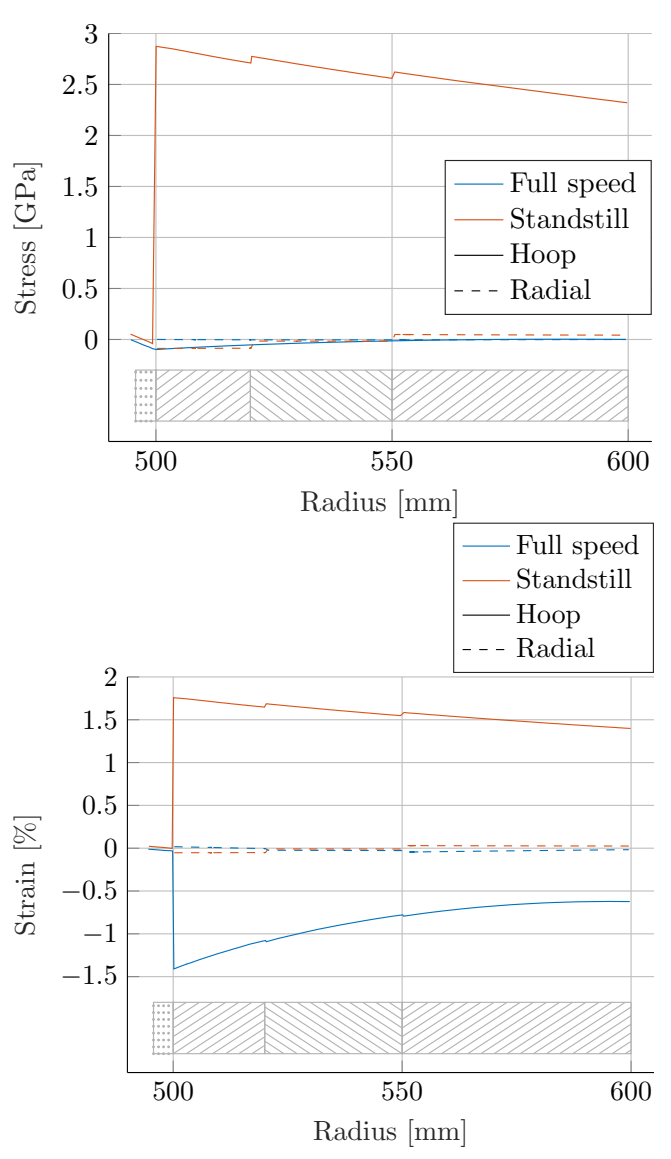

(a)

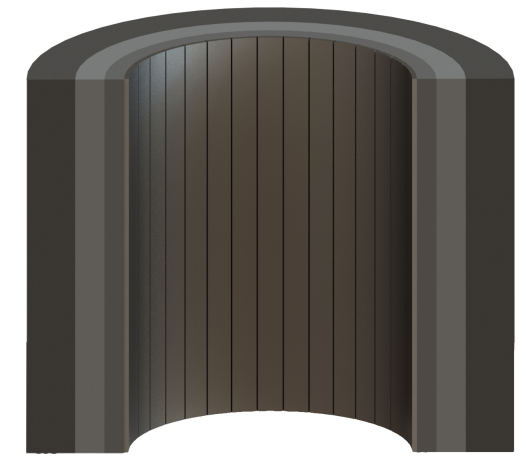

(b)

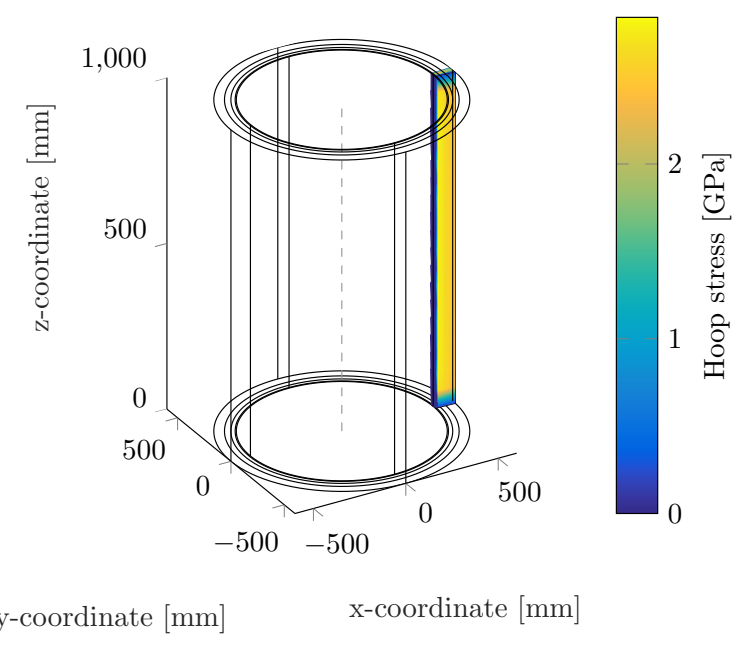

(c)

Figure 1. (a) Stress and strain of proposed design, from the FEA solution. The radial dimensions of the flywheel are illustrated at the bottom. The hatch pattern corresponds to composite material. The dotted pattern is steel. The materials are close to their respective stress and strain-limits; (b) concept drawing of rotor (not to scale). Note the lines in the innermost layer, representing slits in the steel; and (c) FEA solution of the hoop stress distribution inside the hollow cylinder. The simulated $5^{\circ}$ segment is shown. 
Note that carbon composites can expand well in excess of $1.0 \%$, far surpassing the strain limit of magnetic materials, as listed in Table 1. Most materials cannot even sustain the fatigue limit of the unidirectional composite material, which for epoxy matrices is $0.6 \%$ [33]. Any material mounted on the inside of a hollow cylinder will be pressed by centrifugal forces against an expanding composite shell.

Table 1. Material data on materials [34]. Note that the last column assumes linear elasticity, which is an approximation close to Ultimate Tensile Strength (UTS).

\begin{tabular}{cccc}
\hline Material & UTS (MPa) & Elastic Modulus (GPa) & $\epsilon_{\text {max }}(\%)$ \\
\hline Hard Ferrite & 50 & 170 & 0.03 \\
AlNiCo & 50 & 150 & 0.03 \\
NeDyFeB & 75 & 160 & 0.05 \\
SmCo & 35 & 150 & 0.02 \\
Steel E470 & 650 & 210 & 0.31 \\
Sandvik Safeni 52 Steel & 950 & 200 & 0.47 \\
Stahlbecker Spring Steel 1.1274 & 2000 & 206 & 0.97 \\
Copper & 210 & 128 & 0.16 \\
Aluminum 7075-T6 & 572 & 71 & 0.80 \\
\hline
\end{tabular}

This problem can be alleviated by placing the materials on low-friction sleds [31]. Furthermore, a low-modulus plastic matrix can be put between sensitive materials to keep the effects from centrifugal forces compressive. The cycling fatigue behavior is not investigated, but could pose a problem. Another alternative is to infuse epoxy with magnetic materials, in exchange for a decrease of material functionality (a relative permeability $\mu_{r}=13$ ) [1]. Further, there has been some attempts at making flexible magnets [27,35]. In this paper, a reluctance machine solution is proposed, since these can be made from strong steel, which is tolerant to straining and bending.

\section{Switched Reluctance Machine}

The SRM is considered a mechanically robust and relatively efficient topology, and its market penetration is increasing [36]. The SRM has been considered for flywheel energy storage, for example in grid stabilization [37,38], in islanding operation [39] and power smoothing [40]. An SRM can be built with only $\mu$-metals, and the lack of exotic permanent magnet material makes them robust, fault tolerant and capable of high temperature operation while still achieving high torque [41].

Many different topologies with different characteristics are found in the literature. Especially relevant for the hollow cylinders presented above are the external rotor variants [42]. C-core stators have been proposed to simplify the winding process, allowing for simpler manufacturing processes [43]. Although reduced torque ripple is not important for the flywheel rotor itself (since these generally have a high moment of inertia), secondary benefits include decreased losses, decreased vibration and more constant charging and discharging rates. The characteristic ripple torque of axial flux SRMs has been a subject under study [44-46].

Based on the results in the literature, we propose an axial flux outer rotor machine for the hollow cylinder flywheel application, in an effort to avoid salient features and a long flux path in the back yoke. This is since the thickness of the steel layer on the inside of the flywheel was seen to be a major limiting factor above. The electric machine has c-core stators and two axial air gaps, similar to [47], but with an inner stator and fewer rotor poles.

\subsection{Machine Topology}

A 3D schematic view of the proposed axial flux switched reluctance machine is shown in Figure 2. The orange windings (seen in the figure) can be magnetized, driving an axial magnetic field in the stator, drawn in blue. At the top and bottom of the stator, the field flows radially, and then axially, into the air gap. The rotor (drawn in red) flux is axial. Note that the machine laminations are not stacked in the same plane, although all electrical steel can be magnetized in a unipolar fashion. The rotor presented 
in the schematic drawing consists of one pole of laminated (in the $r \varphi$-plane) steel spanning $180^{\circ}$ and a counterweight made of non-ferromagnetic stainless steel placed on the opposite side (not drawn). Note that more poles can be added in the circumferential direction, to change the torque/electrical frequency ratio. The stator is laminated in the $r z$-plane and divided into three phases spaced $120^{\circ}$ apart, allowing for more constant torque. The rotor can also be separated axially to increase torque (while also increasing reluctance due to a larger effective air gap). The schematic drawing above contains two axial poles and, thus, four axial air gaps. A 3-1-2 (\# of poles for the stator, circumferential rotors and axial rotors, respectively) topology as seen in Figure 2 has the minimum amount of circumferential poles.

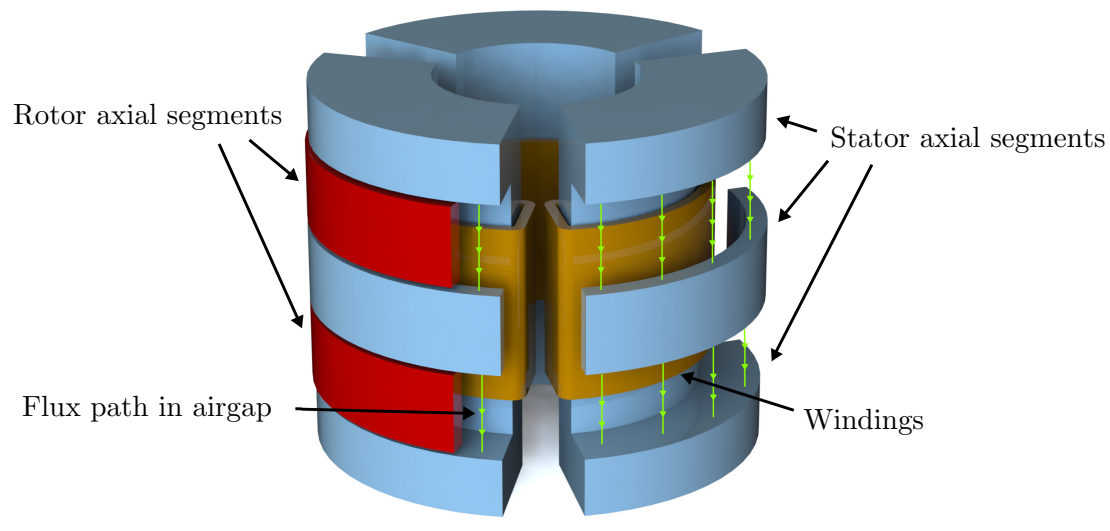

Figure 2. Schematic view of the proposed axial flux machine. The stator is drawn in blue, with orange windings, and the rotor is drawn in red. Only electromagnetically-active material is included in the picture. The concept drawing has three stator poles with separate winding phases and one $180^{\circ}$ rotor pole. The rotor is split into two axial segments. The magnetic flux lines in the air gap are drawn in green.

There are a number of qualitative properties of this topology of interest: Since the air gap is axial, radial expansion during full speed will not affect the magnetic field path. The radial forces in a radial flux machine can be considerable and can affect the rotor fatigue characteristics with respect to cyclic loading. The proposed design has a weak stabilizing force in the radial direction. However, the rotor experiences axial negative stiffness around the air gap. This electric machine topology is intended to be mounted on a hollow flywheel with a set of separate magnetic bearings (which are needed to handle the high relative velocities on the inside of the rim). The axial negative stiffness from the electric machine must then be negated by axial magnetic bearings.

Losses

The high speeds of flywheel rotors require operation in a vacuum to reduce the effects of turbulent drag losses [48]. With no convection or conductive heat transfer, it is hard to transport excess heat away. Black-body radiation is then one of the few mechanisms removing excess heat.

With higher rotational speeds, the frequency dependent losses become increasingly important. Both the rotor and stator can be operated with unipolar magnetization, decreasing the HB-curve area and, in turn, reducing losses. The unipolar control strategy has been previously shown to decrease losses for switched reluctance machines [49].

Since the electric machine can be completely demagnetized, the standby losses can be kept low, decreasing the flywheel spin down rate.

The proposed geometry inherently has plateaus where $\frac{d L}{d \theta}=0$ during a revolution and in turn, no back-emf, which decreases reactive power requirements on the power electronics [50]. 


\subsection{Prototype Construction}

A proof of concept prototype (from hereon referred to as the prototype, in contrast to the full-scale machine analyzed in Section 2) of the electric machine has been designed and built. The prototype has a single circumferential and axial pole in the rotor and three stator poles. One important objective of the prototype is to validate its basic electromagnetic functionality and also gather experiences from the mechanical assembly of this complex geometry, as presented in this paper. Regular bearings were used instead of magnetic bearings, and a composite shell was not fitted onto the rotor.

\subsubsection{Rotor}

The prototype rotor mainly consists of an outer aluminum (7075-T6) cylinder with electrical- and stainless-steel laminations, each $0.5 \mathrm{~mm}$ thick, covering the inside of the cylinder, seen in Figure $3 \mathrm{a}$. Approximately half of the cylinders' inner circumference is covered with electric-steel (M350-50A) [51] and the other half with stainless-steel (X5CrNi18-10) [52] (non-magnetic, acting as a counterweight). The steel densities differed slightly, so the amount of counterweight steel needed was determined by weighing during assembly. Not shown in Figure 3a is the central steel shaft, connected to the rotor through an aluminum disk. The disk has a large external thread with a matching thread found on the inside of the rotor cylinder. The central shaft itself is press-fitted into a hole in the middle of the aluminum disk.

In order to keep the steel laminations in place, two wedges were placed in between the two lamination stacks. These wedges are fastened in the main aluminum cylinder by two screws, creating a stress concentration in the aluminum rotor (which can be three-times larger than the average stress of adjacent materials [53]).

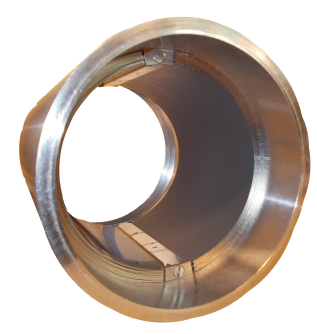

(a)

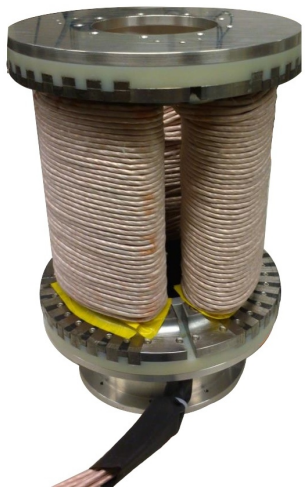

(b)

Figure 3. Photographs of rotor and stator of SRM prototype. (a) The assembled outer-rotor prototype, not including the central shaft. One half ferromagnetic and one half austenitic stainless steel; and (b) the three-phase stator with windings. The aluminum lid is on top, with a plastic plate separating it from the standing laminated c-shaped bits. The windings are wound around each of the three groups of c-shaped bits. The stator is hollow to allow for a shaft in the prototype.

\subsubsection{Stator}

The stator is placed on a base centering it axially and radially inside the rotor, with the pedestal resting on the support structure. In order to make the stator structure rigid, it has a central cylinder with three rectangular holes cut out $120^{\circ}$ apart, making room for the copper windings to wrap around. On the top, there is an aluminum lid and an isolating plastic plate both connected to the central cylinder; an identical plastic isolation plate can also be found on the bottom of the stator connected to the pedestal. Since the stator laminations are stacked in the $r z$-plane, the density of iron will decrease 
with radius. To alleviate this, the lamination stacks are separated with small ferromagnetic wedges. These wedges help to keep the lamination stacks in place, but come with the drawback of increased eddy-current losses, as they are not laminated. Two larger ferromagnetic wedges are placed to decrease the dead-angle in between each phase, allowing the machine to produce a more constant torque while providing space for the copper windings to wrap around. Both of these wedges can be seen in Figure $3 \mathrm{~b}$ alongside the outer radius where the base meets the stator. Also seen in Figure $3 \mathrm{~b}$ are the three stator windings along the length of the stator, one around each stator segment. The main dimensions are presented in Table 2.

Table 2. Dimensions of the prototype.

\begin{tabular}{cc}
\hline Parameter & Value \\
\hline Rotor outer radius, $b$ & $89 \mathrm{~mm}$ \\
Rotor inner radius, $a$ & $84 \mathrm{~mm}$ \\
Rotor height, $h$ & $148 \mathrm{~mm}$ \\
Air gap, $d_{u, l}$ & $1 \mathrm{~mm}$ \\
Stator inner radius, $e$ & $60 \mathrm{~mm}$ \\
Stator outer radius, $f$ & $87 \mathrm{~mm}$ \\
Winding turns $/$ phase, $N$ & 120 \\
\# of phases & 3 \\
\hline
\end{tabular}

\subsubsection{Support Structure}

The support structure keeps the rotor and stator in place while allowing for easy access to the interior of the machine. The structure consists of six steel square pipes linking two large aluminum plates together. In the center of each plate is a central hole where the bearing housings reside. The bearings used here are SKF self-aligning ball bearings (SKF, Göteborg, Sweden) that allow for a slight misalignment of the central axis without affecting the performance of the bearings, reducing the tolerances needed, reducing manufacturing costs and simplifying assembly.

\subsection{Machine Parameters}

\subsubsection{Analytical Model}

Here, we present a simple analytical model for the prototype, to provide some insight on its operation. The relevant variables are presented in Figure 4. A cylindrical coordinate system is used. Let $d$ and $q$ represent solutions when the rotor is aligned and unaligned, respectively, and let indexes $u$ and $l$ denote upper and lower.

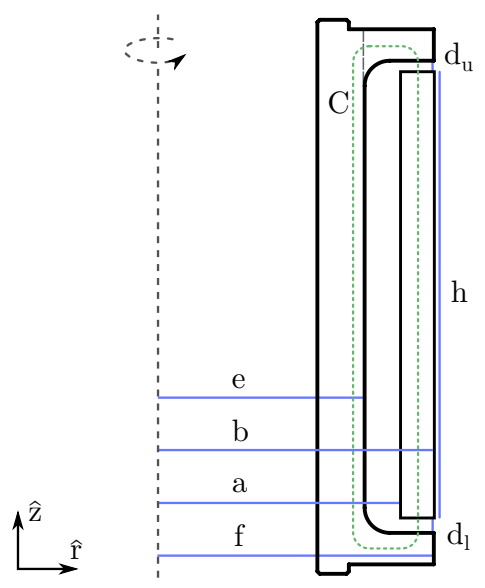

Figure 4. Definition of variables in the $r z$-plane. Note that the curve $C$ lies in the main flux path. 
Begin by considering Ampere's law around curve $C$, as drawn in Figure 4. Assume the magnetic field is constant along the path of integration and that the steel is linear, isotropic, homogeneous and of high relative permeability. Then:

$$
\oint_{C} \mathbf{H} \cdot d \mathbf{s}=N I \Rightarrow\left\{\begin{array}{l}
B_{d}=\frac{\mu_{0} N I}{d_{u}+d_{l}} \\
B_{q}=\frac{\mu_{0} N I}{d_{u}+h+d_{l}}
\end{array},\right.
$$

where $B$ is the magnetic flux density, $N$ is the number of winding-turns and $I$ is the winding current.

The system energy is thus (assume that the $q$-axis field spreads out uniformly between the protruding stator ends):

$$
U=\int \frac{B^{2}}{2 \mu_{0}} d V=\frac{\mu_{0}}{2} N^{2} I^{2}\left(\frac{V_{d}+V_{\text {iron }}}{\left(d_{u}+d_{l}\right)^{2}}+\frac{V_{q}+V_{\text {iron }}}{\left(d_{u}+h+d_{l}\right)^{2}}\right),
$$

where $V_{d, q}$ and $V_{\text {iron }}$ is the volume occupied by a magnetic field in the air gap. The last two factors account for radial and angular displacement,

$$
\left\{\begin{array}{l}
V_{d}=\left(d_{u}+d_{l}\right) \pi\left(b^{2}-a^{2}\right)\left(1-\frac{|\rho|}{b-a}\right)\left(\frac{\frac{2 \pi}{3}-\theta}{2 \pi}\right) \\
V_{q}=\left(d_{u}+h+d_{l}\right) \pi\left(f^{2}-e^{2}\right) 1\left(\frac{\theta}{2 \pi}\right)
\end{array}\right.
$$

where $r \in[a-b, b-a]$ and $\theta \in[0,2 \pi / 3]$. The gradient of the degrees of freedom ( $r$ and $\theta)$ yields the force and torque on the rotor:

$$
\left\{\begin{array}{l}
F_{r}=-\frac{\partial U}{\partial \rho}=\frac{\mu_{0}}{4} N^{2} I^{2} \frac{(b+a)\left(\frac{2 \pi}{3}-\theta\right)}{d_{u}+l_{l}} \frac{|\rho|}{\rho} \\
\tau_{\theta}=-\frac{\partial U}{\partial \theta}=\frac{\mu_{0}}{4} N^{2} I^{2}\left(\frac{b^{2}-a^{2}}{d_{u}+d_{l}}\left(1-\frac{|\rho|}{b-a}\right)-\frac{f^{2}-e^{2}}{d_{u}+h+d_{l}}\right)
\end{array},\right.
$$

The axial force cannot be derived under the assumption of straight field lines. However, there will be negative stiffness around the point when $d_{u}=d_{l}$. That point is an unstable equilibrium. To properly balance this force, we suggest magnetic bearings. The force equations also suggest that the radial position is in a stable equilibrium with positive stiffness around perfect radial alignment (the argument for radial stiffness is overly simplified, but there will be a radially stabilizing force regardless).

The inductance (when radially and axially aligned) can be computed as (where $A$ is area and $\Phi$ magnetic flux):

$$
L=\frac{N \Phi}{I}=\frac{N}{I}\left(\Phi_{d}+\Phi_{q}\right)=\frac{N}{I}\left(B_{d} A_{d}+B_{q} A_{q}\right),
$$

which:

$$
\Longrightarrow\left\{\begin{array}{l}
L_{d}=\mu_{0} N^{2} \frac{\pi}{3} \frac{b^{2}-a^{2}}{d_{u}+d_{l}} \\
L_{q}=\mu_{0} N^{2} \frac{\pi}{3} \frac{f^{2}-e^{2}}{d_{u}+h+d_{l}}
\end{array} .\right.
$$

\subsubsection{Finite Element Analysis}

The prototype electric machine has been simulated in the commercial FEM package COMSOL Multiphysics (COMSOL, Burlington, MA, USA). A 3D geometry has been simulated with nonlinear steel. Figure 5 shows the magnetic flux distribution through a cut section of the prototype.

Prototype inductance was computed via the magnetic energy. However, the magnetic energy for steel with BH-curves is not readily available from an FEM field solution. Therefore, the energy was numerically integrated with the definition [54],

$$
U=\int_{\mathbb{R}^{3}} \int_{0}^{B} \mathbf{H} \cdot d \mathbf{B} d V
$$


Simulations were performed with the magnetizing current I stepped from 0 to $20 \mathrm{~A}$ in steps of $1 \mathrm{~A}$, and the solutions for the $\mathbf{B}$ and $\mathbf{H}$-fields, as well as the volume elements for each mesh point were extracted. These were numerically integrated to attain the inductance of the motor from a simulation with nonlinear steel. The inductance for nonlinear steel was computed at $5 \mathrm{~A}$.

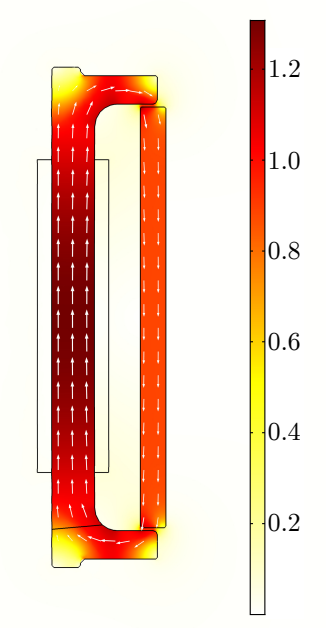

Figure 5. FEM solution of the magnetic flux density at $18 \mathrm{~A}$, for the prototype.

\subsubsection{Experimental Inductance Measurement}

The inductance of the prototype was measured at low voltage/currents with an Agilent U1732A (Agilent Technologies, Santa Clara, CA, USA), at aligned and unaligned rotor position. The behavior at higher currents and steel saturation was obtained with system identification methods. By analysis of voltage and current measurements, as seen in Figure 6, an $L(\theta, I)$ map could be produced.

Data Analysis

The measured data were analyzed by a system identification method derived in this section. The following definition was used for inductance $L$ (to account for nonlinearity):

$$
L(\theta, I)=\frac{d \Phi}{d I}=\frac{d \Phi}{d t} \frac{d t}{d I}
$$

where $\Phi$ is magnetic flux.

The prototype rotor was held fixed for each angle that was measured. $L$ was assumed constant during measurement, partly due to the fact that the rotor was fixed, but also because the current ripple was assumed small enough that a linear relation between flux and current exists in that region. The magnetically-induced potential difference is thus:

$$
v(t)=\frac{d \Phi(t)}{d t}=L(i(t), \theta) \frac{d i(t)}{d t}+i(t) \frac{d L(i(t), \theta)}{d t} \approx L\left(I_{\text {mean }}, \theta_{\text {fixed }}\right) \frac{d i}{d t} .
$$

The circuit equation for one phase is then:

$$
L\left(I_{\text {mean }}, \theta_{\text {fixed }}\right) \frac{d i}{d t}=V_{\text {in }}-R i-V_{\text {diode }}
$$

The current ripple amplitude $\Delta I$ for each period was extracted from the measurement data, as well as the switching period $\Delta T$. These were used to approximate the current derivative:

$$
L\left(I_{\text {mean }}, \theta_{\text {fixed }}\right)=\frac{\Delta T}{\Delta I}\left(V_{\text {in }}-R I_{\text {mean }}-V_{\text {diode }}\right),
$$


Equation (20) can be applied to every pair of points in a large dataset of measurements, and the inductance at each point is then computed with a least squares method.

When $\frac{d I}{d t} \neq 0$, eddy currents are induced that affect the measurements, which is a source of error with this technique.

\section{Measurement Equipment}

Current was measured with a Hall effect sensor (LEM LA 55-P, LEM, Zürich, Switzerland) with a bandwidth of $200 \mathrm{kHz}$ [55]. Voltage was also measured via the Hall effect (LEM LV 25-P), with a 90\% step response time of $40 \mu$ s [56].

The measurement signal was converted to a noise-resistant current signal and fed from the transducers to a 10-bit ADC-unit. Each ADC-conversion took $4 \mu$ s, with a $120 \mathrm{ppm}$ accuracy at a bandwidth of $370 \mathrm{kHz}$ [57]. The analog input aliasing filter was bypassed due to a low noise level during testing, as seen in Figure 6.

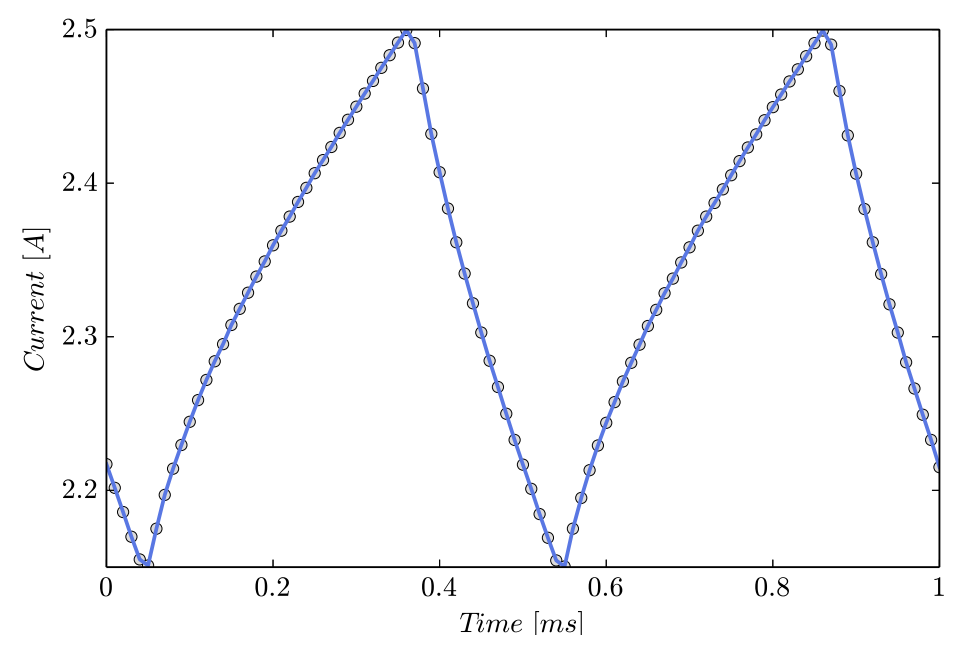

Figure 6. The $100 \mathrm{kHz}$ unfiltered current measurement used for estimating the saturation behavior of the prototype electric machine.

The switching frequency of the current controller was lowered to $2 \mathrm{kHz}$ (from $20 \mathrm{kHz}$ ) for the measurements to increase signal amplitude and increase data point density on the rising and decreasing slopes, as seen in Figure 6. The sample rate was $100 \mathrm{kHz}$. The current and voltage waveform measured in the winding was fed asynchronously through a high-speed first-in-first-out (FIFO) buffer from the measurement FPGA to a measurement data server for analysis.

A separate H-bridge was used to control the current, as seen in Figure 7. The current controller and sampling were done by a CompactRIO programmable automation controller (National Instruments, Austin, TX, USA). The custom circuit boards mounted on the CompactRIO were used to convert current signals into voltage signals. 


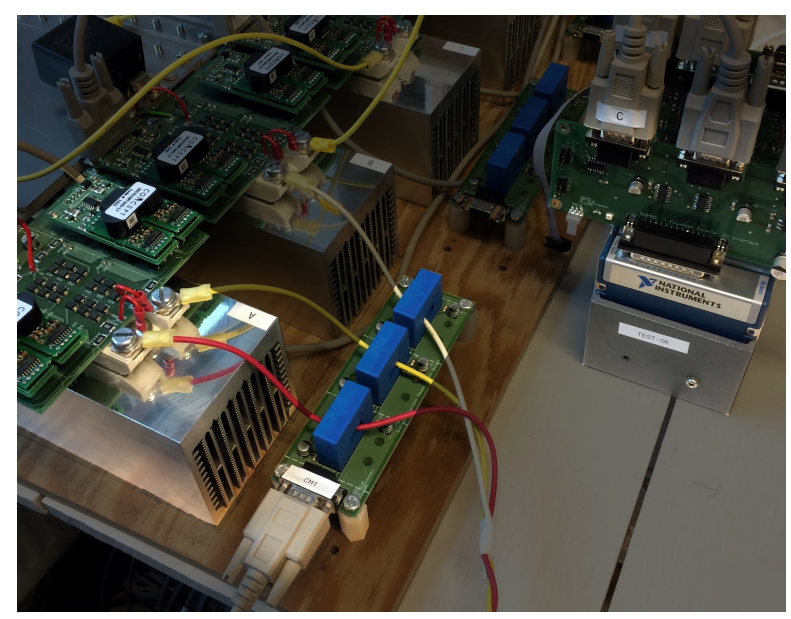

Figure 7. Photo of the single general purpose H-bridge used for driving the currents for system identification measurements, highlighted to the left. The current measurement board is seen in the middle, and the CompactRIO programmable automation controller used for sampling and storing of data is seen to the right.

\section{Results}

For each point in Figure 8b, 2000 switching periods were recorded per angle and current. The prototype rotor was held fixed at 12 different angles, and the current was measured in steps of $1 \mathrm{~A}$ from $0 \mathrm{~A}$ to $20 \mathrm{~A}$. The result of different inductance estimations can be seen in Figure $8 \mathrm{a}$.

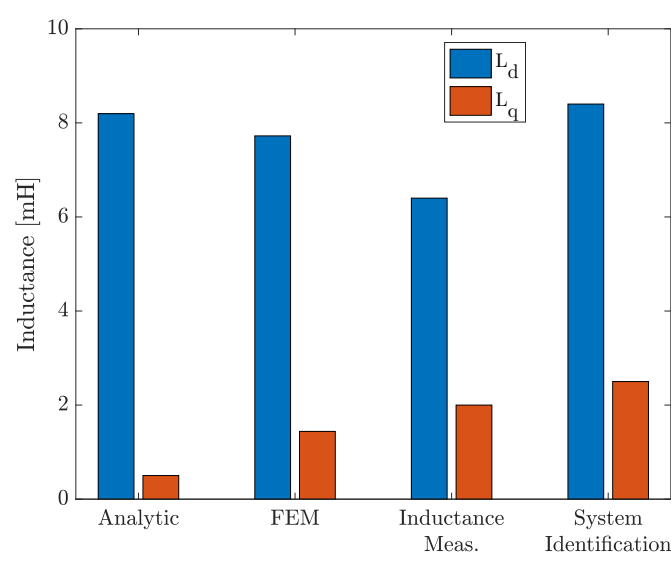

(a)

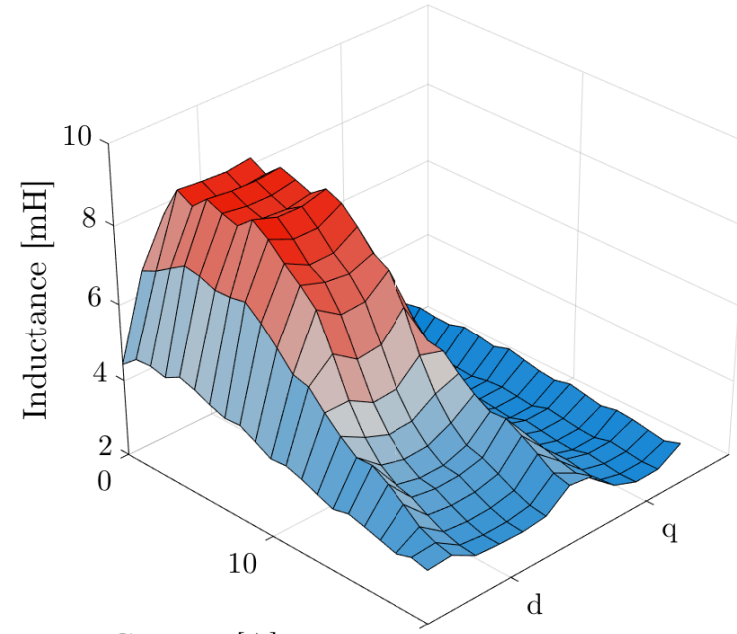

Current $[\mathrm{A}]$
20

(b)

Figure 8. In (a), the $L_{d}, L_{q}$ as retrieved from five different methods are presented: analytical, 3D FEM with linear steel, 3D FEM with nonlinear steel, inductance meter, inductance estimation from $v$ and $i$. A relatively low magnetization was used for the nonlinear FEM computation $(I=5 \mathrm{~A})$, and the difference from the linear FEM value depends on local saturation points. In (b), a map of $L(\theta, I)$ is shown.

\section{Dynamic Simulation}

A dynamic simulation was performed in MATLAB Simulink (Mathworks, Natick, MA, USA) in order to estimate performance during higher speeds than the small prototype presented above can sustain. A flux-based state model for each phase was used to describe the system, as seen in Figure 9. 
The $L_{d}, L_{q}$ inductances estimated above were used as inputs to the simulation, together with the saturation behavior found during system identification, to produce lookup tables for the simulation.
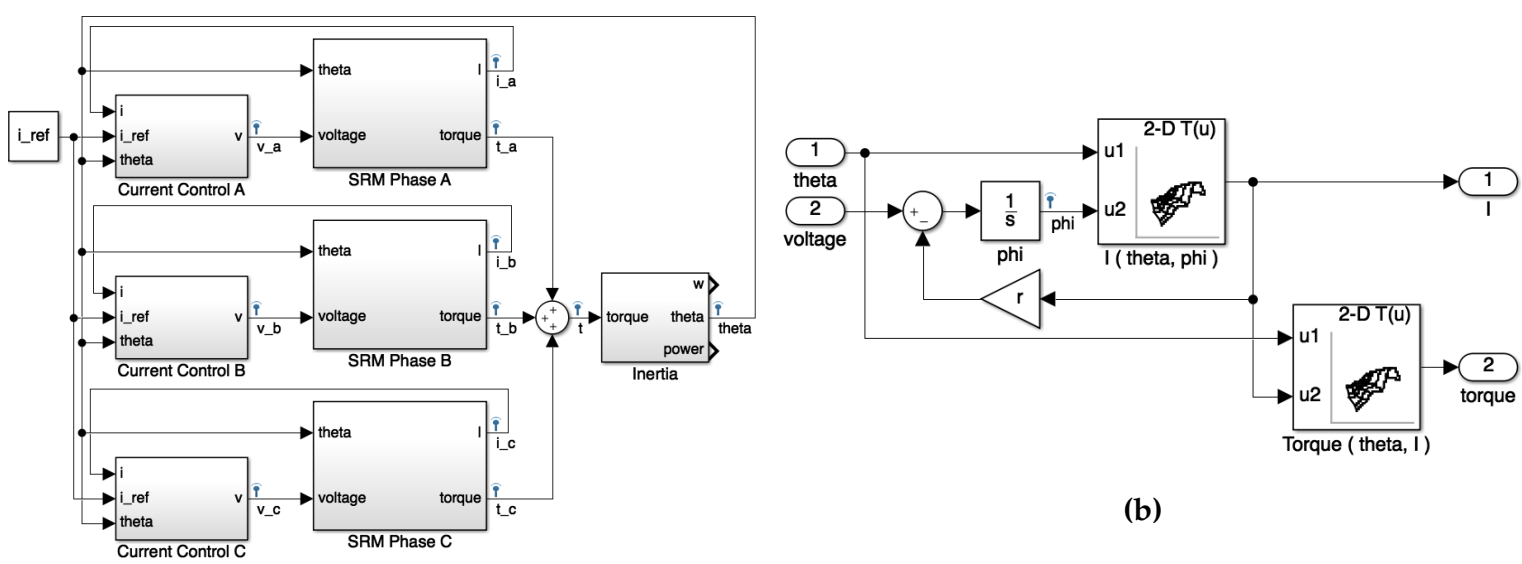

(b)

(a)

Figure 9. Simulink model of SRM. (a) Main model; and (b) sub-model of SRM Phase A.

Two simulations were performed, the first one with the prototype presented above and, secondly, one for the full-scale machine presented in Section 2. For the full-scale machine presented in Section 2, the maximum simulated rotational frequency was found to be $334 \mathrm{~Hz}$, which was used for the simulation below. The simulated voltage and current for one phase during motoring in the prototype is seen in Figure 10.

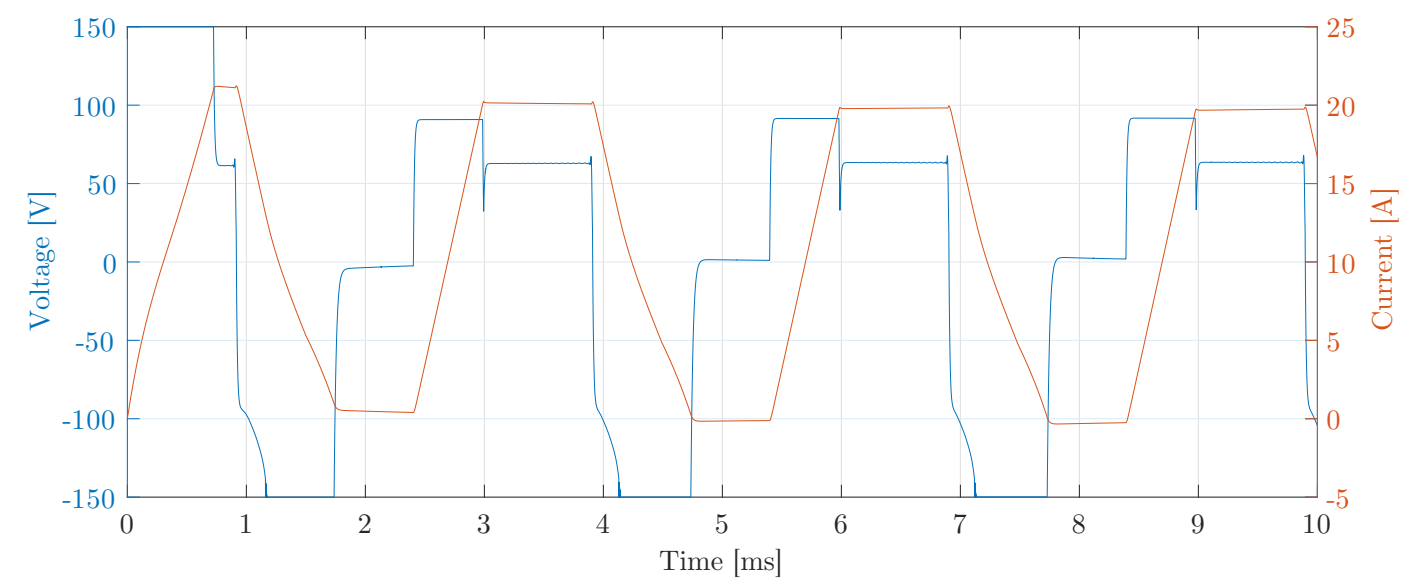

Figure 10. Dynamic simulation showing phase currents and voltages for a single phase, for the prototype. The rotational frequency was $334 \mathrm{~Hz}$.

\section{Discussion}

The mechanical analysis was performed as a two-step process. Firstly a feasible solution in terms of stress was found with the help of the analytical equations, and secondly, this solution was verified in FEA. The full-scale machine was simulated with a $5^{\circ}$ segmentation. For ease of construction, the prototype was constructed with $180^{\circ}$ segments. Ideally, the rotor flux segments would be in the form of stacked wires, shaped for the optimal fill factor. These would then be stacked parallel to the axial flux path. The electric machine behavior would then be altered, due to flux tending to move less tangentially in the rotor. 
The 3D flux path in the proposed topology implies 3D FEM solutions, which demand much in terms of computing power due to the large meshes required. Saturation behavior is also very important in SR machines, implying that a nonlinear material solver is needed, as well. In order to optimize the electrical properties of the machine, a faster approximation would need to be developed and verified.

The analytic solution presented did provide acceptable results for the geometry chosen, although underestimating leakage flux (manifested via a low $L_{q}$ ). It is likely that for an optimized design, in which for example, axial rotor segments are used, or a substantially shorter axial flux path in general, the analytical solutions will deviate more due to unmodeled leakage fluxes.

The practical construction of the prototype machine revealed a few important details. It was found to be hard to have laminated steel through all of the flux path, especially on the stator side of the air gap. A better solution than to use solid steel wedges could be to mount rings on the stator stacked in the $\varphi z$-plane; especially since some of the flux must move tangentially there. The winding process was found to simple and straightforward. Fixing the rotor laminations in place with wedges (as seen in Figure 3a) required special manufacturing, which should be optimized. If using axially-oriented wires as suggested above, an epoxy matrix or similar would probably be beneficial.

The measurements in Figure $8 \mathrm{~b}$ seem to contain high saturation regions of decreasing inductance around $L_{d}$, which would imply negative torque. However, the actual torque is not negative, so the valley-shape is attributed to some physical loss-related effect and/or errors in the estimation assumptions, whose analysis is outside the scope of this paper. Most probably though, the effect is due to some of the smaller components in the stator not being laminated, in which eddy-currents are being induced. The effect can be seen in Figure 6, where the current seems to rise faster just after a switch.

The dynamical simulation showed that the machine does have inductance plateaus, in which there is no back-emf. These plateaus can decrease the reactive power requirements of the power electronics, by allowing for the current to settle. The saturation behavior was included in the model, but no torque ripple or harmonic distortion was modeled. Although the system identification was evaluated at 240 different operating points, the angular resolution was deemed too low to model these properties satisfactorily.

A high peripheral velocity is not commonly optimized in electric machines. However, for an electric machine mounted close to the periphery of a flywheel energy storage, maximal peripheral velocity is directly related to the specific energy of the machine. The full-scale machine is seen to exhibit a large radius and high peripheral velocity when evaluated close to UTS.

The inductance map can be used to compute mean mechanical power $P$ given speed $\omega$ using the following relations:

$$
P=\tau_{\text {mean }} \omega,
$$

where $\tau_{\text {mean }}$ is the mean torque developed when the rotor is passing from the $d$ to the $q$ position. The instantaneous torque can be found by differentiating the co-energy $W^{\prime}$ [50]:

$$
\tau_{\text {mean }}=\text { mean }\left(\frac{\partial W^{\prime}}{\partial \theta}\right)_{\text {I constant }}=\frac{\Delta W^{\prime}}{\Delta \theta}=\frac{\int_{i_{1}=0}^{i_{1}=I} \Phi_{d}-\Phi_{q} d i_{1}}{\theta_{d}-\theta_{q}},
$$

The flux is substituted by integration of Equation (17), which yields:

$$
P=\omega \frac{\int_{i_{1}=0}^{i_{1}=I} \int_{i_{2}=0}^{i_{2}=i_{1}} L\left(\theta_{d}, i_{2}\right)-L\left(\theta_{q}, i_{2}\right) d i_{2} d i_{1}}{\theta_{d}-\theta_{q}},
$$

For the full-scale machine, a larger radius allows for larger winding area. A basic estimate of the full-scale machine rating can be extrapolated assuming all factors scale linearly. For example, assume the stator and rotor poles were tripled, as well as the winding area (assuming volume and sufficient cooling was available) and that the axial length of the rotor was increased and split into ten axial segments, with a ten-fold increase of parallelly-wound windings. Under the assumptions 
above, the power level of the full-scale topology would reach $50 \mathrm{~kW}$. Within the scope of this work, some important aspects have been excluded, such as iron, eddy-current and copper losses, as well as heat transport. The power was also computed close to the UTS, which in a practical implementation is not feasible due to impurities in the composite fibers, as well as containment vessel restrictions (application-dependent).

Future work should include high-speed testing of this topology, in a full-scale flywheel energy storage machine, including magnetic bearings, a vacuum containment system and a carbon composite shell. Before that, the design should be optimized for an axial segmentation, and torque ripple should be investigated and consequently minimized. The flux path should be improved so that no unlaminated steel is magnetized.

\section{Conclusions}

A hollow cylinder flywheel energy storage rotor design was presented, with an ultimate rotor specific energy of $181 \mathrm{Wh} / \mathrm{kg}$ (evaluated close to UTS). This rotor includes electromagnetically-active material in the form of steel, unlike the $195 \mathrm{Wh} / \mathrm{kg}$ record rotor [28], which was accelerated with an air turbine. This value compares favorably to other high-end flywheels, such as the Flybrid Systems Formula 1 flywheel rotor, which had a specific energy of $22.2 \mathrm{Wh} / \mathrm{kg}$ (although not ultimate specific energy). The mechanical analysis of the flywheel was performed with an analytical formulation assuming planar stress in concentric shells of orthotropic (unidirectionally tangentially wound) carbon composites, which was then verified by 3D FEM simulations.

A novel thin outer-rotor switched reluctance machine was proposed and presented, intended to be mounted on the inside of a hollow cylinder flywheel. Numerical analysis evaluated close to UTS found that the electric machine could theoretically reach rim speeds of $1050 \mathrm{~m} / \mathrm{s}$ for the electromagnetically-active material (the rim speed of the outermost composite shell was higher). High rim speed is especially relevant for flywheel energy storage with shaft-less rotors, when the focus is high specific energy. The motor topology is thus an enabling factor for hollow flywheel designs, which does not require shaft-rim connections and which may have lower cost than other designs.

A small-scale prototype was constructed, and the electrical properties were measured experimentally and verified against an analytical model and an FEM simulation. The measurements for this machine can be used as a means of verification for further studies and models of this machine concept, for example when increasing poles (axially and circumferentially). The prototype rotor consisted of one laminated pole covering $180^{\circ}$, leading flux in the axial direction, through axial air gaps into c-shaped stator cores, wound into three different phases. The axial air gaps lead to smaller radial forces (compared to a radial flux machine), and axial forces are zero when the rotor is centered perfectly in the axial direction.

Acknowledgments: This work was conducted within the STandUP for Energy strategic research framework and supported by The Swedish Energy Agency.

Author Contributions: Magnus Hedlund wrote the paper, conceived of and designed the machine and performed measurements. Tobias Kamf helped with writing the paper, made the mechanical design of the prototype and assembled it. Juan de Santiago helped with writing the paper. Johan Abrahamsson and Hans Bernhoff held an advisory role.

Conflicts of Interest: The authors declare no conflict of interest.

\section{References}

1. Hedlund, M.; Lundin, J.; de Santiago, J.; Abrahamsson, J.; Bernhoff, H. Flywheel energy storage for automotive applications. Energies 2015, 8, 10636-10663.

2. Dixon, L.; Porche, I.; Kulick, J. Driving Emissions to Zero; Technical Report; RAND Corporation: Santa Monica, CA, USA, 2002.

3. Head, P. Electro-mechanical flywheel technology. In Proceedings of the Investing in Future Transport Conference, London, UK, 16 August 2010. 
4. Nykvist, B.; Nilsson, M. Rapidly falling costs of battery packs for electric vehicles. Nat. Clim. Chang. 2015, 5, 329-332.

5. Lithium-Ion Battery Life; Technical Report; SAFT: Levallois-Perret, France, 2002.

6. Foley, I. Williams Hybrid Power-Flywheel Energy Storage. Presented at the Engine Expo 2013, Messe Stuttgart, Germany, 4-6 June 2013.

7. 125 V Heavy Transportation Module; Datasheet; Maxwell Technologies, Inc.: San Diego, CA, USA, 2015.

8. Genta, G. Kinetic Energy Storage: Theory and Practice of Advanced Flywheel Systems; Butterworth-Heinemann: Oxford, UK, 1985.

9. Bakholdin, D.; Bosley, R.W.; Rosen, H.A.; Grayer, W. Flywheel Rotor with Conical Hub and Methods of Manufacture Therefor. U.S. Patent US5628232 A, 13 May 1997.

10. Waagepetersen, G.B.F. Flywheel. U.S. Patent US5946979 A, 7 September 1999.

11. Swett, D.W. Flywheel with Self-Expanding Hub. U.S. Patent US6014911 A, 18 January 2000.

12. Spears, W.; Polimeno, M.; Ansbigian, D.C.; Hockney, R. Advanced Flywheel Hub and Method. U.S. Patent US20100206126 A1, 19 August 2010.

13. Johnson, W.E. Flywheel. U.S. Patent US1902505 A, 21 March 1933.

14. Flanagan, R.C.; Wong, J.J. Energy Storage Rotor with Flexible Rim Hub. U.S. Patent US4860611 A, 29 August 1989.

15. Bakholdin, D.; Bosley, R.W.; Rosen, H.A.; Pearson, C.C.; Pano, S.B. Hub and cylinder design for flywheel system for mobile energy storage. U.S. Patent US6175172 B1, 16 January 2001.

16. Brackett, N.C. Stiff Metal Hub for an Energy Storage Rotor. U.S. Patent US6817266 B1, 16 November 2004.

17. Friedericy, J.A.; Towgood, D.A. Layered Flywheel with Stress Reducing Construction. U.S. Patent US4481840 A, 13 November 1984.

18. Gregoire, D.J.; Swett, D.W. Flywheel with Expansion-Matched, Self-Balancing Hub. U.S. Patent US5732603 A, 31 March 1998.

19. Friedericy, J.A.; Towgood, D.A. Centre Assemblies for Flywheels, and Flywheels Incorporating such Assemblies. U.S. Patent EP0026570 A1, 8 April 1981.

20. Losee, J.R. Elliptical Flywheel Apparatus. U.S. Patent US4085627 A, 25 April 1978.

21. Han, Y.H.; Jung, S.Y.; Lee, J.P.; Park, B.J.; Park, B.C.; Jeong, N.H.; Sung, T.H. Large Capacity Hollow-Type Flywheel Energy Storage Device. U.S. Patent US8134264 B2, 13 March 2012.

22. Medlicott, P.A.C. Energy Storage Flywheel. U.S. Patent US4821599 A, 18 April 1989.

23. Maass, D. Integral Composite Flywheel Rim and Hub. U.S. Patent US5784926 A, 28 July 1998.

24. Gabrys, C.W.; Simmons, D.G. Flywheel Hub-to-Trim Coupling. U.S. Patent EP1077335 A1, 21 February 2001.

25. Hull, J.R.; Strasik, M.; Mittleider, J.A.; Wilenski, M.S.; Kozar, M.P. Nested-Rotor Open-Core Flywheel. Patent EP2648318 A2, 3 October 2013.

26. Reinventing the flywheel. In Machine design Digital Edition, 11th ed.; Penton Media Inc.: New York, NY, USA, 2011, pp. 50-53.

27. Arseneaux, J. Development of a $100 \mathrm{kWh} / 100 \mathrm{~kW}$ Flywheel Energy Storage Module; Beacon Power: Wilmington, MA, USA, 2010.

28. Takahashi, K.; Kitade, S.; Morita, H. Development of high speed composite flywheel rotors for energy storage systems. Adv. Compos. Mater. 2002, 11, 40-49.

29. Abrahamsson, J.; Bernhoff, H. Magnetic bearings in kinetic energy storage systems for vehicular applications. J. Electr. Syst. 2011, 7, 225-236.

30. Bolund, B.; Bernhoff, H.; Leijon, M. Flywheel energy and power storage systems. Renew. Sustain. Energy Rev. 2007, 11, 235-258.

31. Abrahamsson, J.; Hedlund, M.; Kamf, T.; Bernhoff, H. High-speed kinetic energy buffer: Optimization of composite shell and magnetic bearings. IEEE Trans. Ind. Electron. 2014, 61, 3012-3021.

32. Toray Industries, Inc. T1000G Data Sheet; Toray Industries, Inc.: Tokyo, Japan, 2016.

33. Akshantala, N.V.; Talreja, R. A mechanistic model for fatigue damage evolution in composite laminates. Mech. Mater. 1998, 29, 123-140.

34. Pyrhonen, J.; Jokinen, T.; Hrabovcova, V. Design of Rotating Electrical Machines; John Wiley \& Sons: Hoboken, NJ, USA, 2009.

35. Kozar, M.P.; Hull, J.R.; Mittleider, J.A.; Wilenski, M.S. Flexible Magnet Directional Stiffening Methods. U.S. Patent US8816543 B2, 3 October 2014. 
36. Tanujaya, M.; Lee, D.H.; Ahn, J.W. Design a novel switched reluctance motor for neighborhoods electric vehicle. In Proceedings of the 2011 IEEE 8th International Conference on Power Electronics and ECCE Asia (ICPE ECCE), Seogwipo, Korea, 30 May-3 June 2011; pp. 1674-1681.

37. Andrade, R.D.; Ferreira, A.C.; Sotelo, G.G.; Neto, J.L.S.; Rolim, L.G.B.; Suemitsu, W.I.; Bessa, M.F.; Stephan, R.M.; Nicolsky, R. Voltage sags compensation using a superconducting flywheel energy storage system. IEEE Trans. Appl. Supercond. 2005, 15, 2265-2268.

38. Rolim, L.G.B.; Ferreira, A.C.; Sotelo, G.G.; de Andrade, R. Flywheel generator with switched reluctance machine. In Proceedings of the International Conference on Electrical Machines (ICEM), Bruges, Belgium, 26-28 August 2002.

39. Iglesias, I.J.; Garcia-Tabares, L.; Agudo, A.; Cruz, I.; Arribas, L. Design and simulation of a stand-alone wind-diesel generator with a flywheel energy storage system to supply the required active and reactive power. In Proceedings of the 2000 IEEE 31st Annual Power Electronics Specialists Conference, Galway, Ireland, 23 June 2000; Volume 3, pp. 1381-1386.

40. Cardenas, R.; Pena, R.; Perez, M.; Clare, J.; Asher, G.; Wheeler, P. Power smoothing using a flywheel driven by a switched reluctance machine. IEEE Trans. Ind. Electron. 2006, 53, 1086-1093.

41. Kiyota, K.; Chiba, A. Design of switched reluctance motor competitive to 60-kW IPMSM in third-generation hybrid electric vehicle. IEEE Trans. Ind. Electron. 2012, 48, 2303-2309.

42. Chen, H.; Gu, J.J. Switched reluctance motor drive with external rotor for fan in air conditioner. IEEE/ASME Trans. Mechatron. 2013, 18, 1448-1458.

43. Mao, S.H.; Tsai, M.C. A novel switched reluctance motor with C-core stators. IEEE Trans. Magnet. 2005, 41, 4413-4420.

44. Madhavan, R.; Fernandes, B.G. Performance improvement in the axial flux-segmented rotor-switched reluctance motor. IEEE Trans. Energy Convers. 2014, 29, 641-651.

45. Shibamoto, T.; Nakamura, K.; Goto, H.; Ichinokura, O. A design of axial-gap switched reluctance motor for in-wheel direct-drive EV. In Proceedings of the 2012 XXth International Conference on Electrical Machines (ICEM), Marseille, France, 2-5 September 2012; pp. 1160-1165.

46. Madhavan, R.; Fernandes, B.G. Comparative analysis of axial flux SRM topologies for electric vehicle application. In Proceedings of the 2012 IEEE International Conference on Power Electronics, Drives and Energy Systems (PEDES), Bengaluru, India, 16-19 December 2012; pp. 1-6.

47. Labak, A.; Kar, N.C. Designing and prototyping a novel five-phase pancake-shaped axial-flux SRM for electric vehicle application through dynamic FEA incorporating flux-tube modeling. IEEE Trans. Ind. Appl. 2013, 49, 1276-1288.

48. Abrahamsson, J.; de Oliveira, J.G.; de Santiago, J.; Lundin, J.; Bernhoff, H. On the efficiency of a two-power-level flywheel-based all-electric driveline. Energies 2012, 5, 2794-2817.

49. Liu, X.; Zhu, Z.Q.; Hasegawa, M.; Pride, A.; Deohar, R.; Maruyama, T.; Chen, Z. Performance comparison between unipolar and bipolar excitations in switched reluctance machine with sinusoidal and rectangular waveforms. In Proceedings of the 2011 IEEE Energy Conversion Congress and Exposition (ECCE), Phoenix, AZ, USA, 17-22 September 2011; pp. 1590-1595.

50. Miller, T.J.E. Switched Reluctance Motors and Their Control; Magna Physics, Oxford University Press: Oxford, UK, 1993.

51. Cogent. Electrical Steel Non Oriented Fully Processed; Datasheet; Cogent Power Ltd.: London, UK, 2002.

52. Deutsche Edelstahlwerke Gmbh. Chromium-Nickel Austenitic Stainless Steel; Datasheet; Deutsche Edelstahlwerke Gmbh: Witten, Germany, 2014.

53. Wahl, A.; Beeuwkes, R. Stress concentration produced by holes and notches. Am. Soc. Mech. Eng. Trans. 1934, 56, 617-636.

54. Wangsness, R.K. Electromagnetic Fields; Wiley: Hoboken, NJ, USA, 1986.

55. LEM. Current Transducer LA 55-P; Datasheet; LEM: Zürich, Switzerland, 2015.

56. LEM. Voltage Transducer LV 25-P; Datasheet; LEM: Zürich, Switzerland, 2014.

57. National Instruments. NI 9205 Data Sheet; National Instruments: Austin, TX, USA, 2015.

(C) 2017 by the authors. Licensee MDPI, Basel, Switzerland. This article is an open access article distributed under the terms and conditions of the Creative Commons Attribution (CC BY) license (http:/ / creativecommons.org/licenses/by/4.0/). 